\title{
Post COVID-19 Vaccine Encephalitis and Romboencephalitis: Three Cases Report
}

\author{
Diana Khedr* and Sulaiman Alkhashan \\ Department of neurology, Farwanya hospital, Kuwait
}

Submission: February 14, 2022; Published: February 28, 2022

*Corresponding author: Diana Khedr, Department of neurology, Farwanya hospital, Kuwait

\begin{abstract}
Background: Since introducing the SARS-CoV-2 vaccination, different adverse effects and complications have been linked to the vaccine. Variable neurological complications have been reported after receiving the COVID-19 vaccine, such as acute encephalopathy.

Case presentation: In this report, we describe three cases previously healthy men who developed acute cerebellar and brainstem symptoms with temporal relationship to ChAd0x1 nCov-19, CSF investigations showed lymphocytic pleocytosis. They received five days of $1 \mathrm{gm}$. methylprednisolone with variable response.

Discussion: Extensive workup for different causes of acute encephalopathy, including autoimmune encephalitis, was negative. Also, one patient improved dramatically after receiving methylprednisolone, supporting an immune-mediated mechanism behind his acute presentation. Accordingly, we think the COVID-19 vaccine is the only possible cause of our patient's presentation, giving the temporal relationship and the absence of other risk factors for encephalopathy.

Conclusion: the clinician should be aware of the possible neurological complications of the different COVID-19 vaccines. Further research is needed to clarify the pathophysiology of such complications. Large, pooled data from observational epidemiologic studies are necessary to
\end{abstract} verify causality.

\section{Background}

The global SARS-CoV-2 pandemic severity has led to an unprecedented effort to develop multiple effective vaccines. Due to excellent safety and efficacy data from clinical trials, the ChAdOx1 nCoV-19 vaccine (AZD1222) was approved consisting of a replication-deficient chimpanzee adenoviral vector ChAdOx1, containing the SARS-CoV-2 structural surface glycoprotein antigen (spike protein; nCoV-19) gene [1,2]. Since introducing the SARS$\mathrm{CoV}-2$ vaccination, different adverse effects and complications have been linked to the vaccine. Variable neurological complications have been reported after receiving the COVID-19 vaccine, such as acute encephalopathy [3-5]. In general, vaccinations can cause a strong expression of proinflammatory cytokines and a $\mathrm{T}$ cell response [6,7]. Peripheral proinflammatory cytokines expressed after vaccination are considered to be causal by reaching the brain and partly resulting in neuroinflammation after microglia activation, depending on the immunogenetic background and the innate immune memory [7]. Herein we present a case series of three patients with autoimmune encephalitis related to prior ChAdOx1 nCoV-19 vaccination, previously undescribed.

\section{Informed Consent}

Written informed consent was obtained for all cases
A copy of the written consent is available for review by the Editor of this journal.

\section{Case Presentation}

\section{First Case}

F.J 30-year-old male patient, previously healthy with no history of any psychiatric illness or drug intake, received the second dose of the ChAdOx1 nCov-19 vaccine experiencing typical side effects like fever and malaise. The following days he developed headache, photophobia, vomiting and progressive neurological symptoms including unsteadiness of gait, oscillopsia, speech disturbance (staccato speech), resulting in admission to our hospital 5 days after vaccination. By examination there was bilateral gaze evoked nystagmus, staccato speech, bilateral intention kinetic tremors in both upper limbs with severly impaired finger to nose and heel to knee tests, severly ataxic gait, hyperreflexia with pathological reflexes and positive frontal lobe release signs. There were no meningeal irritation signs, no extraocular muscle impairment, no motor or sensory or other cranial nerve affection. Emergency brain magnetic resonance imaging (MRI) was essentially normal. A lumbar puncture on the day of admission revealed lymphocytic pleocytosis of 150 cell $/ \mathrm{mm}^{3}$ (with normal proteins and 


\section{Open Access Journal of Neurology \& Neurosurgery}

negative virology PCR for HSV, VZV, CMV, EBV negative culture and sensitivity, negative TB PCR and positive oligoclonal bands suggestive of inflammatory process.

The extensive diagnostic workup remained negative, including paraneoplastic screening (chest X-ray, sonography of the abdomen, and serological examinations of serum and cerebrospinal fluid (CSF) examination for panel of autoimmune encephalitis), vitamin B 1, 6, 12 level and vasculitic screening (ESR, ANA, ANCAb,c ), serum ACE level, HIV abs, HCV abs all were negative. EEG done and was normal. Based on a temporal relationship with the vaccine and after exclusion of all possible etiologies for acquired cerebellar ataxia, possible post COVID-19 vaccination autoimmune encephalitis diagnosis was considered, immunosuppressive therapy with $1 \mathrm{gm}$ methylprednisolone per day for 5 days was initiated with significant improvement of the symptomatology. A follow up lumbar puncture was performed showing decrease in lymphocytic pleocytosis to 6 cells/ $\mu$ l. At discharge, there was still mild ataxic signs. 2 months later developed the previous symptoms again gradually till he became bed ridden from ataxia with evident true bulbar palsy symptoms and dysphagia for fluids. At examination he showed absent gag and palatal reflexes, positive nasal tonation, ataxic flaccid dysarthria, with the previous cerebellar symptoms. There was no motor, sensory, sphinteric, other cranial nerve affection and cognitive affection at all. No history of seizure activity or disturbed conscious level or evident behavioral changes. Lumbar puncture was done with normal results, MRI brain was done for follow up and surprisingly was normal also (Figure 1), pulse methylprednisolone $1 \mathrm{gm}$ was given with IVIG $0.4 \mathrm{mg} / \mathrm{kg} /$ day for 5 days followed by oral steroids tapering over 2 months with marked improvement of his cerebellar, speech, and bulbar symptoms.

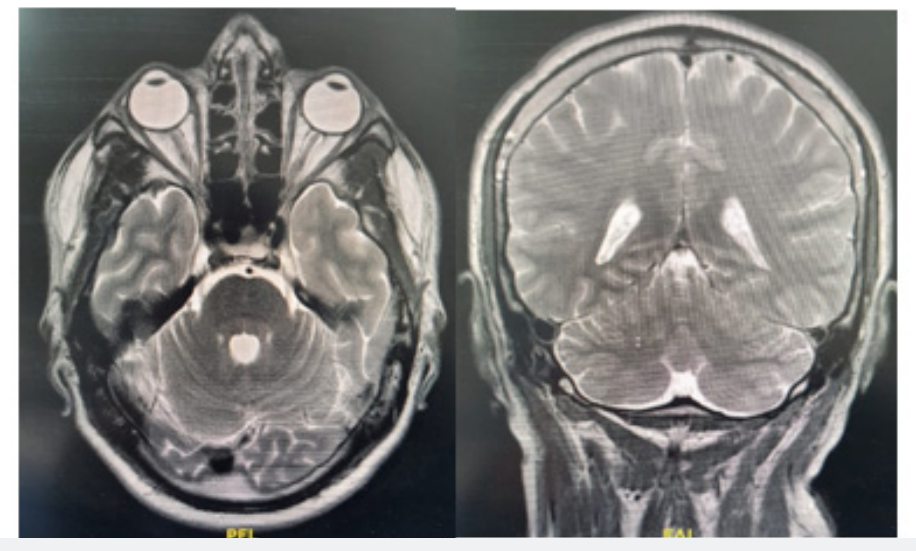

Figure 1: Normal MRI brain of first case.

\section{Second Case}

M.R 39-year-old male previously healthy, had been vaccinated against COVID-19 with ChAdOx1 nCov-19 vaccine, 2 weeks later started to have gradual onset of disorientation and drowsiness, with reported low-grade fever, anorexia and 4 attacks of vomiting.

By examination he was hardly obeying with spontaneous eye opening and inappropriate words, he had bilateral lower motor neuron lesion facial palsy, true bulbar palsy with absent gag reflex, chin drop, no limitation of ocular motility or nystagmus. There was right sided weakness with bilateral sure pyramidal signs (hyperreflexia, sustained ankle clonus and bilateral extensor planters). MRI brain with contrast was done three times during his hospital admission and was normal (Figure 2). Lumbar puncture was done with mild elevated proteins $670 \mathrm{mg} / \mathrm{ml}$ with lymphocytic pleocytosis 130 cell / mm3 with negative virology PCR and positive oligoclonal bands in CSF. Further extensive workup was done including EEG showed background slowing with no specific epileptiform discharge. PET scan was done and was normal, panel for autoimmune encephalitis, virology screen (HIV, HCV, HBV), sarcidosis, lyme disease screening were done and were negative. Anti ganglioside antibodies and AntiGq1b abs was done and were negative. Working diagnosis was autoimmune brainstem encephalitis and was given $1 \mathrm{gm}$ of pulse methylprednisolone for 5 days with minimal improvement only in his conscious level that he became responding to orders but with complete anarthria. Then we offered to him 5 days of IVIG with no additional benefit, even he received one dose of rituximab 1 gm with no effect on his condition. He was planned for CT guided brain biopsy but unfortunately, he developed cardiac arrest and died within 1 month from admission.

\section{Third case}

A.M, 38yrs old male patient previously healthy, 5 days after first dose of AstraZeneca Covid-19 vaccine, developed gradual onset progressive course of douple vision, ataxia, slurred speech, 


\section{Open Access Journal of Neurology \& Neurosurgery}

right sided diminution of hearing, left side drooping of upper eyelid, photophobia and weight loss due to severe anorexia. By examination there was evident ataxic signs in both upper and lower limbs, bilateral horizontal gaze evoked nystagmus, left ptosis, limitation of ocular motility with limited adduction on left side with upper motor neuron signs in the form of hyperreflexia and upgoing planters bilaterally. MRI brain was done showing hyperintense inflammatory signals in cerebellum and brainstem (Figure 3). Lumbar puncture was done, and all parameters were normal with negative oligoclonal bands. Extensive workup was done including PET scan and was normal, panel of autoimmune encephalitis was normal, Aquaborin 4 abs was negative, Antimog abs was negative. CT guided brain biopsy from inflammatory lesion was done showing $\mathrm{T}$ cell infiltration with no B cells at all mainly with active microglia and astrocytes with no evidence of demyelination. Patient received 1 gm methylprednisolone for 5 days and IVIG for 5 days, tocilizumab and rituximab but developed anaphylaxis from it so wasn't completed. He showed slight early improvement in his clinical picture after steroids then unexplained rapid deterioration in his conscious level, respiratory embarrassment and was intubated, then unfortunately, brainstem death within almost three months from onset of his symptom.
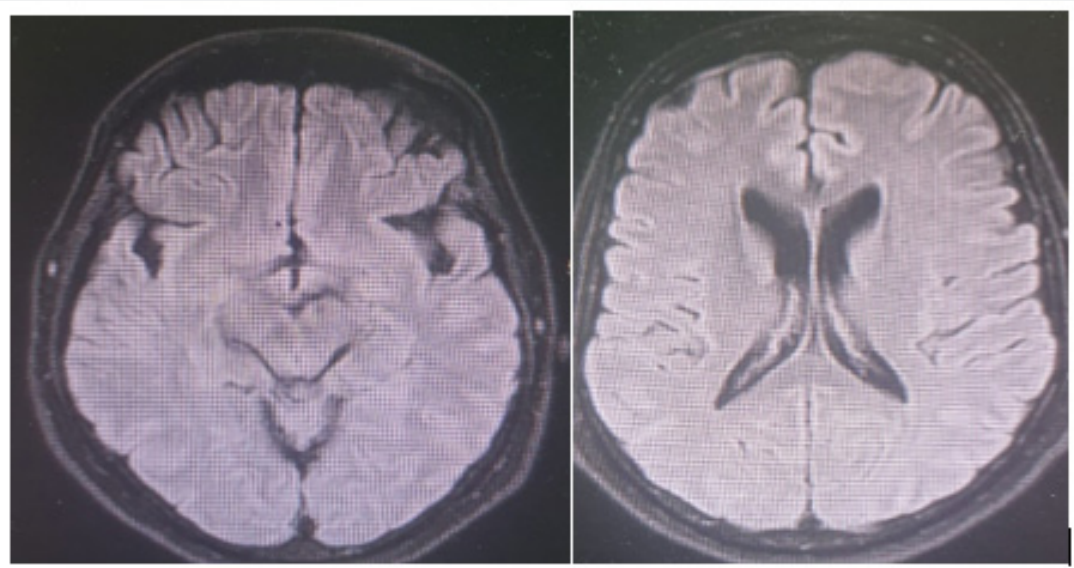

Figure 2: Normal MRI brain of the second case.

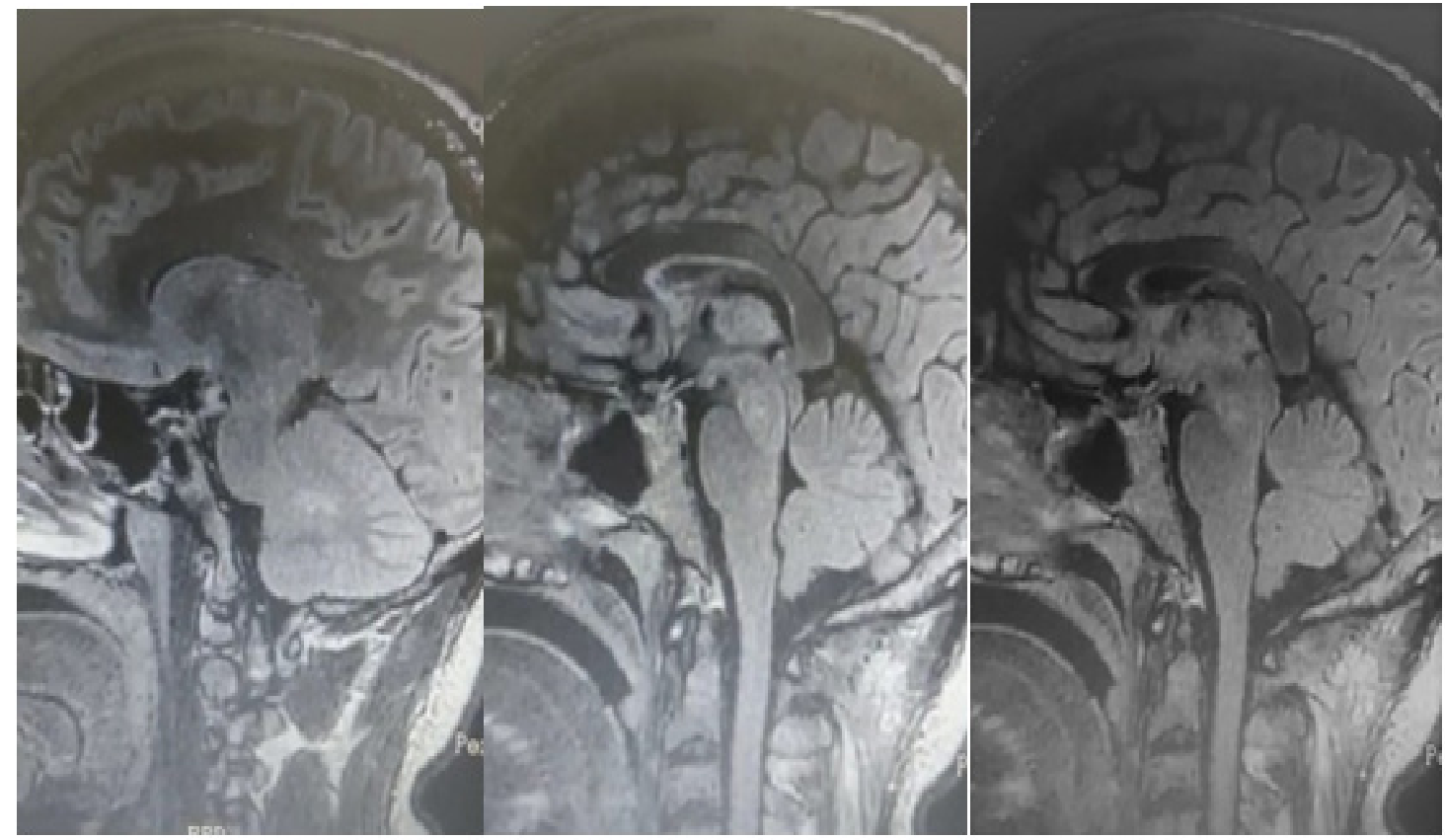

Figure 3: Showing MRI brain of the third case with hyper intense flair signals in midbrain and cerebellar vermis. 


\section{Discussion}

The presented case series is characterized by onset of symptoms of encephalitis within 2 to 14 days after ChAdOx1 nCoV-19 vaccination. In the three cases, the criteria for possible autoimmune brainstem encephalitis including (1) subacute of brainstem and cerebellar signs, (2) CSF pleocytosis, and (3) exclusion of alternative causes was positive. Consequently, the diagnosis of possible autoimmune brainstem encephalitis was made and after exclusion of pathogen-induced encephalitis immunosuppressive treatment was initiated. Fortunately, the first case who was diagnosed early with early introduction of steroids responded to therapy and exhibited a benign course of the disease with almost complete recovery from neurological symptoms. In general, vaccinations can cause a strong expression of proinflammatory cytokines and a $\mathrm{T}$ cell response. This was also demonstrated for ChAdOx1 nCoV-19 vaccine 8 . After vaccination, antigens are recognized as potential pathogens by both conserved pathogen- and damage-associated molecular patterns as well as pattern-recognition receptors that are found on local or peripheral circulating immune cells and on resident stromal cells [7]. Induction and transcription of many target genes occurs, resulting in synthesis and release of pyrogenic cytokines (ie interleukin [IL]-1, IL-6, tumor necrosis factor-alpha [TNF- $\alpha$ ], and prostaglandin-E2 into the bloodstream that mimic the response to natural infection. Thus, a cytokine-mediated inflammatory process is proposed as the key pathophysiological mechanism for COVID-19-related encephalopathy, known as cytokine stormassociated encephalopathy [6-8].

Due to the lack of antibody detection, the diagnosis of a definite autoimmune encephalitis cannot be made. However, based on dramatic improvement after receiving methylprednisolone in the first case, supporting an immune-mediated mechanism behind his acute presentation. Accordingly, we think the COVID-19 vaccine is the only possible cause of our patient's presentation, giving the temporal relationship with the vaccine and the absence of other risk factors for encephalopathy. Our cases report nearly resembles a recently reported cases by Zuhorn et al. [8], L. Baldelli et al. [9], Takata et al. [10] and Rinaldi et al. [11] that describes the occurrence of hyperactive acute encephalopathy post ChAdOx1 nCoV-19 vaccine. In a survey over 20 years in the United States [12] 1,396 cases of encephalitis occurred after vaccination. Responsible vaccinations were hepatitis B (354 cases), influenza vaccination (208 cases), vaccination for measles, mumps and rubella-MMR (208 cases), and vaccination for Haemophilus influenza type B (120 cases). The onset of encephalitis within 2 weeks after vaccination was reported in 708 patients $(50.7 \%)$. Public institutions collect the occurrence of adverse reactions for the different COVID-19 vaccines. Recent data from the National Institute of Public Health of Québec (INSPQ) show that 67 reports of unusual clinical manifestations were made in Quebec for each
100,000 doses administered, all vaccines combined. In the case of AstraZeneca, this proportion rises to 182.5 reports per 100,000 doses. The vast majority of unusual clinical manifestations listed by the INSPQ are considered as without gravity [13]. The occurrence of encephalitis after vaccination with ChAdOx1 nCoV19 (AZD1222) is reported in several publicly available databases. A total of 79 cases of encephalitis worldwide were identified after ChAdOx1 nCov-19 vaccination. By the reported periods and vaccinations given in this period, an estimation of the incidence can be made, which is about 8 per 10 million vaccination doses.

The same analysis yielded 20 reported cases of unexplained encephalitis following vaccination with the Pfizer-Biontech mRNA vaccine (BNT162b2), for a total of more than 110 million doses of vaccine. The estimated incidence is thus 2 in 10 million, and thus only on equarter of case numbers as compared to ChAdOx1 nCov19. The Paul Ehrlich Institute in Germany reported the occurrence of encephalitis within a period of up to 91 days after vaccination with ChAdOx1 nCov-19 [14]. According to the determined incidence, approximately 19 cases with encephalitis of unexplained etiology could be expected per 10 million doses during this period of 91 days [14]. In the survey of 1,396 encephalitis cases over 20 years in the United States, 14 an onset within 2 weeks after vaccination is quite common (50.7\%)-as also seen in our cases. Therefore, within 2 weeks, 2.9 cases of unexplained encephalitis could be expected to occur per 10 million doses of vaccination according to the large UK survey [15]. So, although we are aware, that proof of causality will not be possible in individual cases, we believe the remarkable temporal association between ChAdOx1 nCov-19 vaccination and the presentation of encephalitis in three cases is noteworthy.

\section{Conclusion}

We report here three cases of postvaccinal brainstem encephalitis fulfilling the criteria of possible autoimmune process, in temporal correlation shortly after vaccination with ChAdOx1 nCov-19. The diagnosis was supported by:

i. The temporal association between vaccination and symptom onset,

\section{ii. The characteristic symptoms previously described \\ iii. The extensive exclusion of other etiologies (Table 1), and \\ iv. The response to early immunosuppressive therapy with} corticosteroids. So, we report these cases for increasing awareness for possible autoimmune brainstem encephalitis post COVID-19 vaccination for urgent intervention with immunosuppressive therapy after exclusion of infectious process gives excellent outcome. Obviously, the complication of autoimmune encephalitis after ChAdOx1 nCoV-19 vaccination appears to be very rare. Clearly, the benefit of vaccination outweighs the risks. 


\section{Open Access Journal of Neurology \& Neurosurgery}

Table 1: Summary of demographics, clinical picture, investigations and management in the three cases.

\begin{tabular}{|c|c|c|c|}
\hline & First case & Second case & Third case \\
\hline Age/ sex & $30 / \mathbf{M}$ & $38 / M$ & 39 / M \\
\hline Clinical syndrome & $\begin{array}{l}\text { Cerebellobulbar symp- } \\
\text { toms }\end{array}$ & Brainstem symptoms & Brainstem symptoms \\
\hline $\begin{array}{l}\text { Temporal relation to } \\
\text { COVID-19 vaccine }\end{array}$ & 2 days after second dose & 2 weeks after second dose & 5 days after first dose \\
\hline Standard CSF analysis & $\begin{array}{l}\text { Lymphoctic pleocytosis } \\
150 \text { cell } / \mathrm{mm}^{3}\end{array}$ & $\begin{array}{l}\text { Lymphocytic pleocytosis } \\
130 \text { cell } / \mathrm{mm}^{3}\end{array}$ & $\begin{array}{l}\text { Normal cell count } \\
\text { less than } 5 \text { cell } / \mathrm{mm}^{3}\end{array}$ \\
\hline $\begin{array}{c}\text { Extensive CSF virology PCR (HSV, VZV, } \\
\text { CMV, EPV, Enteroviruses) }\end{array}$ & Negative & Negative & Negative \\
\hline $\begin{array}{l}\text { Panel for autoimmune encephalitis } \\
\text { (serum and CSF) (anti NMDA abs, anti } \\
\text { CASPR, anti LGI1 , anti AMPA1, anti } \\
\text { AMPA2, anti GABA b) }\end{array}$ & Negative & Negative & Negative \\
\hline MRI brain with contrast & Normal & Normal & $\begin{array}{c}\text { Hyperintense inflammatory } \\
\text { t2 and flair signals in cerebellum and } \\
\text { midbrain }\end{array}$ \\
\hline Paraneoplastic screening & Negative & Negative & Negative \\
\hline Offered treatment & $\begin{array}{c}5 \text { days Methylprednis- } \\
\text { olone } \\
\text { and IVIG }\end{array}$ & $\begin{array}{l}5 \text { days Methylprednisolone, } \\
\text { IVIG and rituximab }\end{array}$ & $\begin{array}{l}5 \text { days Methylprednisolone, } \\
\text { IVIG, PLEX, tocilizumab and ritux- } \\
\text { imab }\end{array}$ \\
\hline Fate and prognosis & $\begin{array}{l}\text { Marked improvement } \\
\text { and } \\
\text { discharge on oral } \\
\text { steroids }\end{array}$ & Death after 2 months from onset & Death after 3 months of onset \\
\hline
\end{tabular}

\section{References}

1. COVID-19 Map. Johns Hopkins Coronavirus Resource Center.

2. Voysey M, Clemens SAC, Madhi SA, Lily Y Weckx, Pedro M Folegatti, et al. (2021) Safety and efficacy of the ChAdOx1 nCoV-19 vaccine (AZD1222) against SARS-CoV-2: an interim analysis of four randomised controlled trials in Brazil, South Africa, and the UK. Lancet 397:99-111.

3. Greinacher A, Thiele T, Warkentin TE, Karin Weisser, Paul A Kyrle, et al. (2021) Thrombotic thrombocytopenia after ChAdOx1 nCov-19 vaccination. N Engl J Med 384(22): 2092-2101.

4. Graf T, Thiele T, Klingebiel R, Greinacher A, Schäbitz W-R, et al. (2021) Immediate high-dose intravenous immunoglobulins followed by direct thrombin-inhibitor treatment is crucial for survival in SarsCovid-19-adenoviral vector vaccine-induced immune thrombotic thrombocytopenia VITT with cerebral sinus venous and portal vein thrombosis. J Neurol 268(12): 4483-4485.

5. Schultz NH, Sørvoll IH, Michelsen AE, Ludvig A Munthe, Fridtjof Lund-
Johansen, et al. (2021) Thrombosis and thrombocytopenia after ChAdOx1 nCoV-19 vaccination. N Engl J Med 384(22): 2124-2130.

6. Giannotta G, Giannotta N (2018) Vaccines and neuroinflammation. Int J Pub Heal Safe 3:1000163.

7. Hervé C, Laupèze B, Del Giudice G, Arnaud M Didierlaurent, Fernanda Tavares Da Silva, et al. (2019) The how's and what's of vaccine reactogenicity. NPJ Vaccines 4: 39.

8. L Baldelli, G Amore, A Montini, I Panzera, S Rossi, et al. (2021) Hyperacute reversible encephalopathy related to cytokine storm following COVID-19 vaccine. J Neuroimmunol 358: 577661.

9. Frédéric Zuhorn, Tilmann Graf, Randolf Klingebiel, Wolf-Rüdiger Schäbitz, Andreas Rogalewski (2021) Postvaccinal Encephalitis after ChAdOx1 nCov-19. Ann Neurol 90(3): 506-511.

10. Junko Takata, Simon M Durkin, Solomon Wong, Michael S Zandi, Josephine K, et al. (2021) A case report of ChAdOx1 nCoV-19 vaccineassociated encephalitis. BMC Neurol 21(1): 485. 
11. Virginia Rinaldi, Gianmarco Bellucci, Andrea Romano, Alessandro Bozzao, Marco Salvetti (2021) ADEM after ChAdOx1 nCoV-19 vaccine: A case report. Mult Scler 1-4.

12. Ewer KJ, Barrett JR, Belij-Rammerstorfer S, Hannah Sharpe, Rebecca Makinson, et al. (2021) T cell and antibody responses induced by a single dose of ChAdOx1 nCoV-19 (AZD1222) vaccine in a phase $1 / 2$ clinical trial. Nat Med 27(2): 270-278.

13. Al Qudah Z, Abukwaik W, Patel H, Souayah N (2021) Encephalitis after vaccination in United States. A report from the CDC/FDA vaccine adverse event reporting system. [1990-2010] (P03.151) Neurology 78: P03.151.
14. Granerod J, Ambrose H, Davies N, Jonathan P Clewley, Amanda L Walsh, et al. (2010) Causes of encephalitis and differences in their clinical presentations in England: a multicentre, population-based prospective study. Lancet Infect Dis 10(12): 835-844.

15. Marquez Loza AM, Holroyd KB, Johnson SA, Marquez Loza AM, Holroyd $\mathrm{KB}$, et al. (2021) Guillain-Barre Syndrome in the Placebo and Active Arms of a COVID-19 Vaccine Clinical Trial: Temporal Associations Do Not Imply Causality. Neurology.

\section{Your next submission with Juniper Publishers} will reach you the below assets

- Quality Editorial service

- Swift Peer Review

- Reprints availability

- E-prints Service

- Manuscript Podcast for convenient understanding

- Global attainment for your research

- Manuscript accessibility in different formats

( Pdf, E-pub, Full Text, Audio)

- Unceasing customer service

Track the below URL for one-step submission

https://juniperpublishers.com/online-submission.php 